
2 Research Square
Preprints are preliminary reports that have not undergone peer review.
They should not be considered conclusive, used to inform clinical practice,
or referenced by the media as validated information.

\title{
TRPM7 Silencing Modulates Glucose Metabolic Reprogramming to Inhibit the Growth of Ovarian Cancer by Enhancing AMPK Activation to Promote HIF-1a Degradation.
}

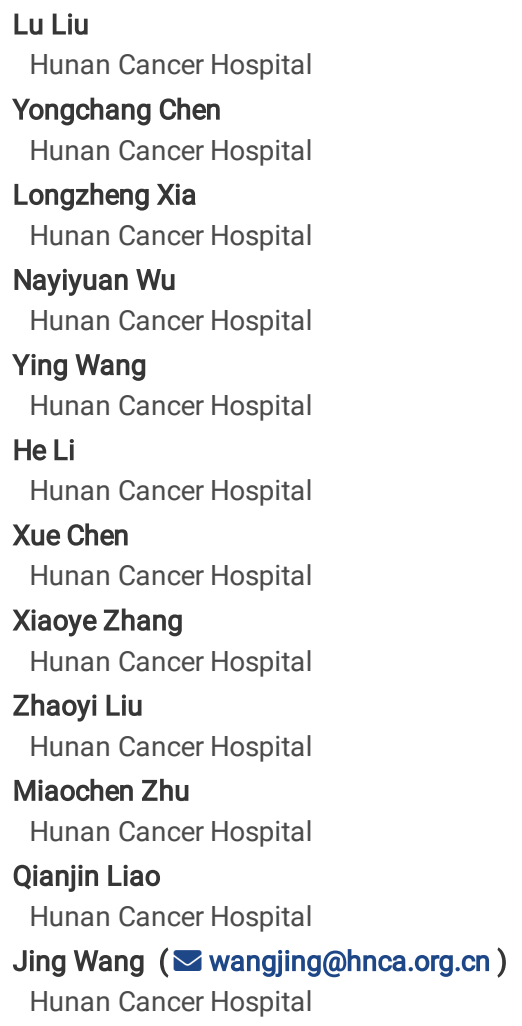

Research

Keywords: TRPM7, AMPK, HIF-1a, Glycolysis, Oxidative phosphorylation, Ubiquitination, Ovarian cancer

Posted Date: July 28th, 2021

DOI: https://doi.org/10.21203/rs.3.rs-752342/v1

License: (9) (7) This work is licensed under a Creative Commons Attribution 4.0 International License. Read Full License 


\section{Abstract}

\section{Background}

Tumor cell metabolic reprogramming is crucial for the malignant behavior of cancer cells by promoting their proliferation. However, little is known on how transient receptor potential 7 (TRPM7) modulates metabolic reprogramming in ovarian cancer.

\section{Methods}

The effects of TRPM7 silencing on transcriptome profile, glucose uptake, lactic acid production, extracellular acidification rate (ECAR), oxygen consumption rate (OCR), intracellular ROS and ATP levels, and NAD+/NADH ratios in ovarian cancer cells were examined. The impacts of TRPM7 silencing on the levels of glycolysis-related HK2, PDK1 and oxidative phosphorylation (OXPHOS)-related IDH3B and UQCRC2, HIF-1a expression and AMPK phosphorylation were determined in ovarian cancer. The effect of AMPK activity on HIF-1a ubiquitination degradation was investigated in ovarian cancer cells.

\section{Results}

Compared with the control, TRPM7 silencing suppressed the proliferation of ovarian cancer cells and promoted their apoptosis by shifting preferable glycolysis to OXPHOS. In parallel, TRPM7 silencing decreased the glucose uptake of tumor-bearing mice and TRPM7 levels were negatively correlated with IDH3B and UQCRC2, but positively with HK2 and PDK1 expression in ovarian cancer tissues. Mechanistically, TRPM7 silencing significantly increased AMPK phosphorylation and decreased HIF-1a protein levels in ovarian cancer, particularly in HIF-1a silencing cells. The shifting from glycolysis to OXPHOS by TRPM7 silencing was abrogated by HIF-1a over-expression and impaired by inhibiting AMPK activity in ovarian cancer cells. Moreover, enhanced AMPK activation inhibited glycolysis, which was abrogated by HIF-1a over-expression in ovarian cancer cells. Moreover, the enhanced AMPK activation promoted HIF-1a ubiquitination degradation.

\section{Conclusions}

TRPM7 silencing enhanced AMPK activation to shift glycolysis to oxidative phosphorylation by promoting HIF-1a ubiquitination degradation in ovarian cancer. Hence, TRPM7 may be a therapeutic target for intervention of ovarian cancer.

\section{Introduction}

Ovarian cancer is a common gynecological malignancy in women. Its prevalence and incidence remain high, affecting many women in the world. Currently, the standard therapies for ovarian cancer include surgical resection and chemotherapy, but ovarian cancer patients still have a 5-year survival rate of < $50 \%$ due to the tumor recurrence and distant metastasis [1-3]. Hence, discovering the molecular pathogenesis, and novel biomarkers and targets is of high significance in prognosis and treatment of ovarian cancer.

It is well known that the majority of healthy cells prefer glucose metabolism by oxidative phosphorylation (OXPHOS) to produce high levels of ATP and reactive oxygen species (ROS), which can cause cell injury and promote apoptosis. In contrast, malignant tumor cells can reprogram their energy metabolism and escape from immune destruction [4,5]. Tumor cells can alter their glucose metabolism by preferring glycolysis even in a normoxic environment to support their malignant behaviors, proliferation, invasion and metastasis, called the "Warburg effect" [6-8]. However, it is still unclear how what factors regulate the Warburg effect and OXPHOS to support the growth of ovarian cancer.

The hypoxia inducible factor (HIF)-1a and adenosine 5'-monophosphate (AMP)-activated protein kinase (AMPK) signal pathways are the major regulators of glycolysis and OXPHOS, and they are crucial for the metabolic reprogramming in tumor cells $[9,10]$. AMPK is an evolutionarily conserved serine / threonine kinase. AMPK can be activated by many factors, such as AMP / ATP and ADP / ATP ratios [11, 12] and intracellular calcium through CaMKK2-mediated phosphorylation [13-15]. HIF-1a is commonly expressed by mammalian cells, but it is rapidly degraded by the intracellular oxygen-dependent ubiquitin protease degradation pathway in a normoxygenic condition. HIF-1a expression is significantly up-regulated and HIF-1a protein stabilizes in a hypoxic condition. The AMPK and HIF pathways can also modulate the expression and function of several transcription factors, such as FoxO, NF-KB, NRF2, and p53, as well as protein kinases and other factors, including mTOR, ULK1, HDAC5, and SIRT1 [16]. However, how little is known on the AMPK and HIF pathways modulate the metabolic reprogramming in ovarian cancer.

Transient receptor potential melastatin 7 (TRPM7) is a unique cation channel protein, and functionally responsible for transportation of cations (such as calcium, magnesium, and others), thereby increasing [Ca2 +]i levels. In addition, TRPM7 has a kinase activity and can activate itself and other substrates, dependent on ATP. TRPM7 expression is up-regulated in a variety of tumors and can enhance malignant behaviors of cancer cells [17]. Our previous study has revealed that up-regulated TRPM7 expression is related to pelvic lymph node metastasis and poor prognosis of human ovarian cancer and inhibition of TRPM7 can suppress the invasion and metastasis of ovarian cancer cells through attenuating the $\mathrm{Ca} 2^{+}-\mathrm{PI} 3 \mathrm{~K} / \mathrm{AKT}$ signaling [18]. Interestingly, inhibition of TRPM7 can promote HIF-1a degradation in prostate cancer cells [19]. Given that glycolysis inhibition is associated with HIF-1a degradation we hypothesize that TRPM7 activation to increase [Ca2 +]i may modulate the HIF-1a/AMPK signaling to regulate glucose metabolism and promote ovarian cancer cell proliferation.

This study investigated the impacts of TRPM7 silencing on ovarian cancer cell proliferation, apoptosis, glucose metabolism and the AMPK / HIF-1a signaling. We found that TRPM7 silencing enhanced OXPHOX, inhibited glucose uptake, glycolysis, lactic acid production and suppressed the proliferation of ovarian cancer cells by enhancing AMPK activation and HIF-1a degradation. Together, our findings indicate that TRPM7 is a novel therapeutic target and may shed new lights on the regulation of metabolic reprogramming and pathogenesis of ovarian cancer. 


\section{Results}

\section{TRPM7 silencing attenuates the growth of ovarian cancer by shifting glucose metabolic reprogramming.}

Given that up-regulated TRPM7 expression is related to poor prognosis of ovarian cancer [17], we determined the impact of TRPM7 expression on cell proliferation. First, we generated TRPM7 stably silencing SKOV3 and H08910 cells (Figure S1A). To understand how TRPM7 silencing regulated gene expression in ovarian cancer cells, we performed RNA-seq analyses between SKOV3 sh-Control and SKOV3 sh-TRPM7 cells. We found that with a fold change of $\geq 2.0$ and $P<0.05$, there were 2873 differentially expressed genes (DEGs) between SKOV3 sh-Control and SKOV3 sh-TRPM7 cells, and among the DEGs, 1222 were up-regulated and 1651 were down-regulated in SKOV3 sh-TRPM7 cells (Fig. 1A, Figure S1B and C), which were supported by the Gene Set Enrichment Analysis (GSEA). The GO and KEGG analyses indicated that the DEGs were involved in the process of cell cycling, positive regulation of mitotic cell cycling and acute inflammatory response in ovarian cancer (Fig. 1B, Table 1). To test the functional outcomes of TRPM7 silencing, we quantified the proliferation of different groups of ovarian cancer cells by the EdU assay. As shown in Fig. 1C, there were less numbers of EdU ${ }^{+}$cells in the TRPM7-silenced SKOV3 or H08910 cells than their sh-control cells. Flow cytometry analysis exhibited that TRPM7 silencing significantly increased the frequency of apoptotic SKOV3 and H08910 cells ( $\mathrm{P}<0.001$, Fig. 1D). Furthermore, TRPM7 silencing significantly decreased the tumor sizes and weights in a mouse xenograft of SKOV3 tumors $(P<0.01$, Fig. 1E). Given that rapid proliferation and metabolic reprogramming are hallmarks of cancer cells, we further performed the $G 0$ analyses and found that the DEGs were enriched in metabolic processes, such as cellular processes, DNA metabolism, macromolecule metabolism, primary metabolism, cellular metabolism, regulation of metabolism, and others (Fig. 1F). It is notable that many DEGs regulated glycolysis and OXPHOS, and they included the glycolysis-related PDK1, HK2, LDHB and OXPHOS-related IDH3B, UQCRC1, UQCRC2. TRPM7 silencing also down-regulated HIF-1a expression in SKOV3 cells (Fig. 1G). Further GSEA analyses revealed the potential signaling pathways, such as the AMPK signal pathway, were enriched in SKOV3 cells (Fig. 1H, Table 2). Accordingly, we speculate that TRPM7 silencing may suppress the proliferation of ovarian cancer cells by shifting metabolic reprogramming.

Table 1

KEGG Pathways and GO annotation enriched in high-risk and low-risk groups using GSEA

\begin{tabular}{|c|c|c|c|c|c|c|c|c|}
\hline NAME & SIZE & ES & NES & $\begin{array}{l}\text { NOM } \\
\text { p-val }\end{array}$ & FDR q-val & $\begin{array}{l}\text { FWER } \\
\text { p-val }\end{array}$ & $\begin{array}{l}\text { Rank } \\
\text { at } \\
\text { max }\end{array}$ & $\begin{array}{l}\text { Le: } \\
\text { ed! }\end{array}$ \\
\hline KEGG_HSA04110_CELL_CYCLE & 116 & -0.4744268 & -1.329925 & 0 & 0.32765228 & 1 & 2757 & $\begin{array}{l}\text { tag } \\
45^{c} \\
\text { list } \\
22 \\
\text { sig } \\
=5\end{array}$ \\
\hline GO_GO:0045931_POSITIVE_REGULATION_OF_MITOTIC_CELL_CYCLE & 121 & -0.4059705 & -1.7015916 & 0 & 0.2011301 & 0.758 & 1579 & $\begin{array}{l}\text { tag } \\
26^{c} \\
\text { list } \\
13^{c} \\
\text { sig } \\
=2\end{array}$ \\
\hline GO_GO:0002526_ACUTE_INFLAMMATORY_RESPONSE & 32 & 0.55722696 & 1.6772016 & 0 & 0.41694513 & 0.837 & 895 & $\begin{array}{l}\text { tag } \\
25 \\
\text { list } \\
7 \% \\
\text { sig } \\
=2\end{array}$ \\
\hline
\end{tabular}

Table 2

KEGG Pathways enriched in high-risk and low-risk groups using GSEA.

\begin{tabular}{|c|c|c|c|c|c|c|c|c|}
\hline NAME & SIZE & ES & NES & $\begin{array}{l}\text { NOM } \\
\text { p-val }\end{array}$ & FDR q-val & $\begin{array}{l}\text { FWER } \\
\text { p-val }\end{array}$ & $\begin{array}{l}\text { Rank } \\
\text { at max }\end{array}$ & Leading edge \\
\hline KEGG_HSA04152_AMPK_SIGNALING_PATHWAY & 95 & 0.2476375 & 1.2687452 & 0 & 0.4344668 & 1 & 1244 & $\begin{array}{l}\text { tags }=14 \%, \text { list }=10 \%, \\
\text { signal }=15 \%\end{array}$ \\
\hline
\end{tabular}

\section{TRPM7 silencing shifts metabolic reprogramming to OXPHOS in ovarian cancer cells}

Tumor cells usually prefer glycolysis to provide necessary energy and metabolites for their proliferation and switching to OXPHOS can promote high levels of ROS production and cell apoptosis [20]. To test whether TRPM7 silencing could shift the glucose metabolic reprogramming, we measured the levels of glucose consumption and lactic acid production in the sh-control and TRPM7 silencing ovarian cancer cells. In comparison with the sh-control cells, TRPM7 silencing significantly reduced the levels of glucose consumption by $>50 \%$ and lactic acid production by $35-40 \%$ in both SKOV3 and $\mathrm{HO} 8910$ cells $(\mathrm{P}<0.05$, $P<0.01$, Figs. $2 A$ and $B$ ). Seahorse assays exhibited that TRPM7 silencing also decreased ECRA, but increased OCR (Fig. 2C and D). TRPM7 silencing also increased the ratios of $\mathrm{NAD}^{+} / \mathrm{NADPH}$ and the levels of ATP and ROS in both SKOV3 and H08910 cells $(\mathrm{P}<0.05, \mathrm{P}<0.01, \mathrm{Fig}$. 2E-G). IHC displayed that carvacrol treatment reduced the expression of TRPM7 in xenograft ovarian tumor tissues in mice (Fig. $2 \mathrm{H}$ ). More importantly, PET-CT imaging revealed that 
carvacrol treatment not only reduced the tumor size, but also attenuated ${ }^{18} \mathrm{~F}$-FDG uptake in tumor-bearing mice by significantly decreasing mean standard uptake value and total volume $(P<0.01$, Fig. 2 l). Collectively, these results indicate that TRPM7 silencing shifts glycolysis to OXPHOS in ovarian cancer, contributing to its inhibition of ovarian cancer growth.

\section{TRPM7 silencing attenuates the glycolysis process and enhances the OXPHOS process of ovarian cancer cells}

To understand how TRPM7 modulated metabolic reprogramming in ovarian cancer, we analyzed the expression of TRPM7, glycolysis-related HK2, PDK1, and OXPHOS-related IDH3B and UQCRC1 in 60 ovarian cancer tissues by IHC (Fig. 3A). The information of those patients is shown in Table S1. Correlation analysis indicated that TRPM7 expression levels in ovarian cancer tissues were positively correlated with HK2 $(P<0.01)$ and PDK1 $(P<0.01)$, but negatively correlated with IDH3B $(\mathrm{p}<0.01)$ and UQCRC1 in this population $(P<0.05$, Fig. 3B), supporting the notion that TRPM7 promotes glycolysis in ovarian cancer. Consequently, TRPM7 silencing decreased the relative HK2 and PDK1 mRNA transcripts, but increased IDH3B and UQCRC1 mRNA transcripts in both SKOV3 and H08910 cells $(P<0.05, P<0.01$, Fig. 3C). Similar patterns of the relative levels of HK2, PDK1, IDH3B and UQCRC1 proteins were detected in these cells by Western blot and immunofluorescence (Fig. 3D and E, Figure S2D). PKM2 is a key enzyme for glycolysis and its nuclear translocation is associated with malignant behaviors of tumor cells [21]. To test the importance of PKM2, we characterized the intracellular distribution of PKM2 and found that TRPM7 silencing obviously decreased the levels of nuclear PKM2, but increased its cytoplasmic PKM2 in both SKOV3 and H08910 cells (Fig. 3F, Figure S2F). Finally, decreased levels of HK2 and PDK1 proteins, but increased levels of IDH3B and UQCRC1 expression were observed in TRPM7-silenced SKOV3 tumors (Fig. 3G). Hence, TRPM7 silencing modulated the levels of glycolysis- and OXPHOS-related enzyme expression to shift glycolysis to OXPHOS in ovarian cancer.

\section{Activation of the AMPK signal pathway inhibits glycolysis that is dependent on high levels of HIF-1a in ovarian cancer cells}

It is well known that AMPK activation can promote OXPHOS and inhibit glycolysis by down-regulating the function of HIF-1a that can promote glycolysis in tumor cells under hypoxic conditions [22-25]. To understand how TRPM7 silencing modulates the HIF-1a and AMPK signaling, we characterized the levels of HIF-1 $a$ and AMPK expression and activation in the indicated ovarian cancer cells by Western blot and IHC. Clearly, TRPM7 silencing elevated the relative levels of AMPK phosphorylation, but minimized the HIF-1a expression in SKOV3 and H08910 cells and SKOV3 tumors (Fig. 4A). Hence, TRPM7 silencing enhanced AMPK activation and reduced HIF-1a protein to switch preferable glycolysis to OXPHOS in ovarian cancer cells. To further understand the importance of AMPK activation in shifting glycolysis to OXPHOS, we tested the effect of altered AMPK activation on the HIF-1a-regulated glycolysis in ovarian cancer cells. We first established stable HIF-1 a silencing SKOV3 and H08910 cells. Compared with the vehicle-treated control cells, treatment with Compound C (CC, an inhibitor of AMPK) significantly inhibited AMPK activation (Figure S3A-B), but increased glucose uptake, lactate acid production and ECAR in both SKOV3 and H08910 cells (Fig. 4B, C and F), which were significantly mitigated or abrogated in the HIF-1a silencing SKOV3 and HO8910 cells. In contrast, treatment with metformin (an activator of AMPK) not only increased AMPK phosphorylation (Figure S3C-D), but also significantly decreased glucose uptake, lactic acid production and ECAR in both SKOV3 and H08910 cells (Fig. 4D, E and G). However, the decreased glycolysis by metformin was abrogated by HIF-1a over-expression in ovarian cancer cells. These two lines of evidence demonstrated that AMPK activation inhibited glycolysis that was dependent on high levels of HIF-1a presence in ovarian cancer.

\section{AMPK activation or HIF-1a over-expression mitigates the TRPM7 silencing-inhibited glycolysis in ovarian cancer cells.}

To understand how HIF-1a and AMPK activation regulate the TRPM7 silencing-induced metabolic reprogramming in ovarian cancer cells, we first generated HIF-1a over-expression in TRPM7 silencing ovarian cancer cells. Second, we tested the impact of TRPM7 silencing on the HIF-1a-mediated glycolysis in SKOV3 and HO8910 cells. Comparison with the control cells, TRPM7 silencing significantly decreased glucose uptake, lactic acid production and ECAR in SKOV3 and H08910 cells, but did not significantly change glucose uptake, lactic acid production and ECAR in the HIF-1a over-expressing SKOV3 and HO8910 cells (Fig. 5A-C). These data indicated that TRPM7 deficiency suppressed glycolysis in ovarian cancer cells, which was abrogated by HIF-1a over-expression. Next, we tested how inhibition of AMPK modulated the TRPM7 silencing-promoted OXPHOS in ovarian cancer cells. While TRPM7 silencing significantly increased the production of ATP, ROS and the ratios of NAD ${ }^{+}$NADPH as well as OCR in ovarian cancer cells, which were significantly mitigated or abrogated by treatment with CC (Fig. 5D-G). Thus, HIF-1a over-expression significantly mitigated or abrogated the TRMP7 silencing-decreased glycolysis and inhibition of AMPK abrogated the TRPM7 silencing-enhanced OXPHOS in ovarian cancer cells.

\section{TRPM7 silencing enhances the AMPK activation and decreases HIF-1a protein levels to shift glycolysis to OXPHOS in ovarian cancer cells.}

Next, we explored how TRPM7 silencing modulates AMPK activation, HIF-1a expression, OXPHOS and glycolysis in ovarian cancer cells. We initially quantified the IDH3B and UQCRC1 expression in the different groups of cells. Relative to the untreated control cells, treatment with metformin to activate AMPK obviously increased IDH3B and UQCRC1 expression, which were mitigated by HIF-1a silencing in SKOV3 and H08910 cells (Fig. 6A, Figure S4A). In contrast, treatment with $\mathrm{CC}$ to inhibit AMPK activation remarkably decreased the relative levels of IDH3B and UQCRC1 expression, which were further reduced in the HIF1a silencing SKOV3 and H08910 cells (Fig. 6B, Figure S4B). Similarly, TRPM7 silencing increased AMPK phosphorylation, IDH3B and UQCRC1 expression in those cells, which were limited by CC (Fig. 6C, Figure S4C). In contrast, TRPM7 silencing reduced the HIF-1a, HK2 and PDK1 expression in SKOV3 and H08910 cells, which were rescued by induction of HIF-1a over-expression (Fig. 6D, Figure S4D). Together, TRPM7 silencing enhances the AMPK activation and decreases HIF-1a protein levels to shift glycolysis to OXPHOS in ovarian cancer cells.

\section{TRPM7 silencing enhances the AMPK activation to promote HIF-1a ubiquitination and degradation in ovarian cancer cells.}

Finally, we investigated how TRPM7 silencing decreased HIF-1a protein in ovarian cancer cells. Hypoxia induced HIF-1a protein expression in ovarian cancer cells, but not in the TRPM7 silencing ovarian cancer cells (Fig. 7A). Treatment with MG132, a proteasome inhibitor, remarkably restored HIF-1a protein levels in ovarian cancer cells regardless of hypoxia condition and TRPM7 silencing (Fig. 7B), indicating that TRPM7 silencing promoted the ubiquitination and proteasomal degradation of HIF-1a. Furthermore, treatment with CC to inhibit AMPK activation restored HIF-1a protein levels in both control and TRPM7 
silencing ovarian cancer cells under hypoxic and normoxic conditions (Fig. 7C and D). Moreover, treatment with CC also decreased the ubiquitination of HIF-1a in both control and TRPM7 silencing ovarian cancer cells under hypoxic and normoxic conditions (Fig. 7E and F). Therefore, TRPM7 silencing enhanced AMPK activation to promote the ubiquitination and proteasomal degradation of HIF-1a, shifting glycolysis to OXPHOX in ovarian cancer cells.

\section{Discussion}

Exploring the potential mechanisms for ovarian cancer growth and finding new therapeutic targets have become a current research hotspot [26]. TRPM7 expression is up-regulated in various tumors $[19,27,28]$. Furthermore, up-regulated TRPM7 expression is positively related to pelvic lymph node metastasis and poor prognosis of ovarian cancer [29]. Inhibition of TRPM7 can minimize the malignant behaviors of ovarian cancer [17, 18]. Here, we reported that TRPM7 silencing changed the transcriptomes involved in modulating cell cycle and acute inflammation, which are linked to tumor cell proliferation and apoptosis [30-32] and modulate the tumor environment [33-36]. Actually, we found that TRMP7 silencing suppressed ovarian cancer cell proliferation and enhanced their apoptosis in vitro and xenograft tumor growth in mice. Hence, TRPM7 acts as an oncogenic factor to promote ovarian cancer growth and is a potential target for development of therapies for ovarian cancer.

It is well known that glucose metabolism by the Warburg effect and OXPHOS can provide nutrients and energy to support the proliferation and biosynthesis in tumor cells $[8,37,38]$. Our results indicated that TRPM7 silencing shifted glycolysis to OXPHOS in ovarian cancer cells. Evidently, TRPM7 silencing significantly minimized glucose uptake, lactic acid production, ECAR, but elevated ATP and ROS levels, NAD+/NADH ratios and OCR in ovarian cancer cells. Consistently, PET-CT imaging revealed that treatment with carvacrol to inhibit TRPM7 activity also decreased 18F-FDG uptake by xenograft ovarian cancer in mice. Furthermore, TRPM7 silencing also decreased the relative levels of glycolysis-related HK2 and PDK1 expression as well as PKM2 nuclear translocation, but increased OXPHOS-related IDH3B and UQCRC1 expression in ovarian cancer cells and tissues. The PKM2 is a specific isoform of pyruvate kinase and a rate-limiting enzyme controlling the glycolysis process. The PKM2 is highly expressed in many types of tumors, including ovarian cancer [39-43]. Studies have shown that PKM2 can translocate to the nucleus, where it forms a complex with HIF-1a and acts as a transcription coactivator of HIF-1a to promote the transcription of HIF-1a targeted genes (such as glycolytic rate-limiting enzymes). Hence, TRPM7 silencing inhibited the proliferation of ovarian cancer cells by shifting from glycolysis to OXPHOS [44-46]. OXPHOS can produce high levels of ATP and ROS, which can induce oxidative inflammation and excessive ROS levels can damage DNA and trigger cell apoptosis [33,36,47]. These may explain why TRPM7 silencing significantly increased the frequency of apoptotic ovarian cancer cells.

Glucose metabolic reprogramming by converting glycolysis to OXPHOS or tumor cell metabolic plasticity has been noticed in several types of tumor cells and is regulated by the HIF-1 $\mathrm{a}$ and AMPK signaling $[9,10,24,25]$. Actually, the enhanced AMPK activation inhibits the expression of HIF-1a and glycolysis-related regulators of HK2, PKM, glucose transporter (GLUT1), fructose-6-phosphate-2-kinase / fructose-2,6-bisphosphatase 4 (PFKFB4) and lactate dehydrogenase A (LDHA) [10,48-50]. Our data revealed that TRPM7 silencing down-regulated HIF-1a expression, but enhanced AMPK activation in ovarian cancer cells, extending previous observations [51-54]. Furthermore, treatment with metformin to enhance AMPK activation promoted the shifting from glycolysis to OXPHOS in ovarian cancer cells, which was abrogated by HIF-1a over-expression. This shifting may supply fewer nucleotides and lipid precursors for macromolecular synthesis in ovarian cancer cells. These, together high levels of ROS to promote the apoptosis of ovarian cancer cells, inhibit ovarian cancer growth. In contrast, treatment with CC to inhibit AMPK activation minimized the shifting from glycolysis to OXPHOS, dependent on high HIF-1a levels in ovarian cancer cells[51, 52, 55,56]. Apparently, the HIF-1a and AMPK signaling has opposite roles in regulating glycolysis and OXPHOS in ovarian cancer. More importantly, we found that TRPM7 silencing promoted HIF-1a ubiquitination and degradation in ovarian cancer cells. It is possible that TRPM7 silencing to reduce $\left[\mathrm{Ca}^{++}\right]$i may activate the AMPK to promote HIF-1a ubiquitination proteasomal degradation that attenuates the HIF-1a-enhanced glycolysis to shift glycolysis to OXPHOS, inhibiting the proliferation of ovarian cancer cells and promoting their apoptosis. Conceivably, these findings may uncover the regulation of the TRPM7/AMPK/HIF-1a axis on the glucose metabolic reprogramming in ovarian cancer.

\section{Conclusion}

Our data indicated that TRPM7 silencing shifted glycolysis to OXPHOS in ovarian cancer cells and attenuated the proliferation of ovarian cancer cells and promoted their apoptosis. Mechanistically, TRPM7 silencing enhanced AMPK activation and promoted HIF-1a ubiquitination and degradation in ovarian cancer cells. Hence, our findings may uncover the new function of TRPM7 in the growth of ovarian cancer and imply that TRPM7 may be a new target for design of therapies for ovarian cancer.

\section{Materials And Methods}

\section{Specific reagents}

The specific reagents induced antibodies against Hexokinase II (ab209847), PDK1 (ab110025), IDH3B (ab247089), TRPM7 (ab109438), UQCRC1 (ab223746), H3 (ab1791), rabbit anti-PKM2 (ab85555), goat anti-rabbit IgG (ab6721) and goat anti-mouse IgG (ab6728, Abcam, Cambridge, USA), phospho-AMPKa (\#2535, Cell Signaling Technology, Saint Louis, USA), Alexa Fluor488-conjugated goat anti-rabbit IgG (\#35552), Alexa Fluor594-conjugated goat anti-mouse IgG (\#35560, Invitrogen, Waltham, USA), compounds of Dorsomorphin (Compound C) $2 \mathrm{HCl}$ (S7306) and metformin $\mathrm{HCl}$ (S1950, Selleckchem, Houston, USA) and DAPI staining solution (C1005, Beyotime, Beijing, China).

\section{Patients}

We recruited 60 ovarian cancer patients without prior radiotherapy and chemotherapy in the Department of Gynecology, Cancer Hospital, Xiangya Medical College of Central South University of China. We collected surgical ovarian specimens when they underwent a surgery for removal of the tumor. Individual 
patients singed the written informed consent. The experiments were approved by the Joint Ethics Committee of Hunan Cancer Hospital and Affiliated Tumor Hospital of Xiangya Medical College of Central South University of China (approval number: KYJJ-2019-001).

\section{Cells and culture}

Human ovarian cancer SKOV3 and H08910 cells were provided by the Cancer Institute, Central South University and they were identified by STR. SKOV3 and H08910 cells were cultured in RPMI 1640 medium containing $10 \%$ fetal bowel serum (FBS, Life Technologies, Carlsbad, USA) at $37^{\circ} \mathrm{C}$ in $5 \% \mathrm{CO}_{2}$. Some cells were cultured in a hypoxic incubator of $1 \% \mathrm{O}_{2}, 5 \% \mathrm{CO}_{2}$, and $94 \% \mathrm{~N}_{2}$ at $37^{\circ} \mathrm{C}$.

\section{Sequencing of mRNAs}

Total RNA was extracted from different groups of SKOV3 sh-control and SKOV3 sh-TRPM7 cells and the samples (1-2 $\mu \mathrm{g} /$ each) were used for generation of libraries using the KAPA Stranded RNA-Seq Library Prep Kit (Illumina), according to the manufacturer's protocol by KangChen BioTech, China. The barcoded libraries were purified and quantified using an Agilent 2100 bioanalyzer. The mixed libraries of different samples were denatured with $0.1 \mathrm{M}$ NAOH, diluted to 8 pM and amplified in situ on TruSeq SR Cluster Kit v3-cBot-HS (\#GD-401-3001, Illumina, USA). The samples were sequenced at $2 \times 150$ bp in an Illumina Xten/NovaSeq sequencer. The levels of gene expression were measured as fragments per kilobase per million reads (FPKM) and the differentially expressed genes (DEGs) were defined when a fold change $\geq 2.0$ and a $P$-value of $<0.05$.

\section{Bioinformatics}

We analyzed the DEGs between the sh-control (C) and sh-TRPM7 (T) groups using Gene Set Enrichment Analysis (GSEA, GSEA v4.0.3 for windows) (http://software.broadinstitute.org/gsea/index.jsp) [57]. First, we analyzed the DEGs in the biological signaling using the MSigDB (Molecular Signatures Database) (http://software.broadinstitute.org/gsea/msigdb) with 1000-rounds. The identified DEGs were defined with a false discovery rate (FDR) < 0.25 and a family-wise error rate (FWR) $<0.05$. Second, the DEGs were subjected to the Gene Ontology (GO) analysis organism (http://www.geneontology.org), particularly in Biological Process, Cellular Component and Molecular Function. The DEGs in the top GO lists were analyzed by Fisher's exact test with a P-value of $\leq 0.05$. Finally, the DEGs were analyzed for their pathways using the KEGG with a $P$-value of $<0.05$.

\section{Immunohistochemistry (IHC)}

The expression levels of specific proteins in ovarian cancer tissues were characterized by IHC using a kit (CW2069, CoWin Biosciences). Briefly, individual paraffin-embedded tissue sections $(3 \mu \mathrm{m})$ were treated with $3 \%$ bowel serum albumin (BSA) and underwent routine-antigen retrieval. The sections were probed with mouse anti-Hexokinase II (1: 500), anti-PDK1 (1: 600), anti-IDH3B (1: 2000), anti-phospho-AMPKa (1: 100), anti-TRPM7 (1: 700), UQCRC1 (1: 400) or control mouse or rabbit IgG at $4^{\circ} \mathrm{C}$ overnight. The sections were cultured with horseradish peroxidase (HRP)-conjugated detection antibodies (1:5000) and reacted with DAB. The sections were counter-stained with hematoxylin. The immune staining signals were photoimaged under a light microscope and semiquantitatively evaluated by two pathologists in a blinded manner, according to the percentages of positively stained cells and the intensity of IHC signals. The percentages of positively stained cells were scored as 0 : $<5 \% ; 1: 6 \%-25 \% ; 2: 26 \%-50 \% ; 3$ : $>50 \%$. The intensity of IHC signals was quantified as 0 : colorless; 1 : light yellow; 2: brown yellow; 3 : dark brown. A final score in each image was achieved as the intensity score $\times$ percentage score and the levels of protein expression were defined as 0 : no expression; $1-4$ : low levels of its expression; 5-9: high levels of its expression.

\section{Transduction and transfection}

The lentiviruses for TRPM7 knockdown, and HIF-1a overexpression or knockdown were purchased from Genepharma (shanghai, China). SKOV3 and H08910 cells $\left(5 \times 10^{5} /\right.$ well) were cultured overnight and transduced with lentivirus at a MOI of 5 for expression of Con-sh, TRPM7-sh1, TRPM7-sh2, TRPM7-sh3, TRPM7-sh4, HIF-1a-sh or HIF-1a. The target sequences are shown in the Supplementary Tables S2. After 48 hours of incubation, their total RNAs and proteins were extracted for verification of gene silencing or over-expression. Some cells were cultured in the presence of G418 (500 $\mu \mathrm{g} / \mathrm{ml}, \mathrm{Biofrox}, \mathrm{Germany})$ for 14 days to generate stable gene silencing or over-expressing cells. Subsequently, we characterized the cell clones by quantitative real-time PCR (qRT-PCR) and Western blot.

\section{Immunofluorescence}

SKOV3-Con-sh, SKOV3-TRPM7-sh, H08910-Con-sh and H08910-TRPM7-sh cells were stained with rabbit anti-Hexokinase II (1: 100), mouse anti-PDK1(1: 100), rabbit anti-IDH3B (1: 100), mouse anti-UQCRC1 (1: 70), rabbit anti-PKM2 (1: 50). Subsequently, they were reacted with Alexa Fluor488-goat anti-rabbit IgG and Alexa Fluor594-goat anti-mouse IgG, and nuclear-stained with DAPI. The cells were examined under a fluorescent microscope.

\section{qRT-PCR}

We extracted total RNAs from each specimen or group of cells using Trizol reagent, and reversely transcribed them into cDNA using Revert Aid First Strand cDNA Synthesis Kit (K1622, Thermoscientific, USA). We quantified the relative levels of target gene to Tubulin mRNA transcripts by qRT-PCR using the FastStart Essential DNA Green Master kit (06924204001, Lifescience, Roche, Mannheim, Germany) and specific primers in the RocheLightCycler ${ }^{\circledR} 96$ instrument and software (05815916001, Lifescience). The sequences of primers are shown in the Supplementary Tables S3. We performed the PCR reactions in triplicate and analyzed the data using the $2^{-\Delta \Delta C t}$ method.

\section{Western blot}


We performed Western blot assays to quantify the relative levels of target protein expression in different groups of cells [58]. Briefly, we performed sodium dodecyl sulfate-polyacrylamide gel electrophoresis (SDS-PAGE) to separate the cell lysate proteins $(30-50 \mu \mathrm{g} / \mathrm{lane}) \mathrm{using} 10 \% \mathrm{gels}$ and transferred them onto polyvinylidene difluoride (PVDF) membranes. We treated the membranes with $5 \%$ BSA in TBST and probed the membranes overnight at $4{ }^{\circ} \mathrm{C}$ with antibodies against HK2, PDK1, IDH3B, UQCRC1, AMPK, phosphor-AMPK, TRPM7 and a-Tubulin. Subsequently, we incubated the membranes with HRP-labeled second antibodies and developed the blots with the ECL substrate (32109, ThermoScientific). Finally, we quantified the relative levels of individual proteins to aTubulin using the ImageJ software (Madison, USA).

\section{Animal experiments and PET/CT study}

We obtained female BALB/c nude mice (6-week-old, $20 \mathrm{~g}$ ) from SLA Laboratory Animal (Changsha, China) and maintained them in a specific pathogen-free room. In the first set of experiments, we injected each mouse subcutaneously with five millions of SKOV3-Con-sh or SKOV3-TRPM7-sh cells in 0.15 mL of saline. Four weeks later, we dissected, weighed, photoimaged the tumors. In another set of experiments, we injected each mouse subcutaneously with $5 \times 10^{6}$ SKOV3 cells. Three weeks later, the experimental group of mice were intraperitoneally injected $50 \mathrm{mg} / \mathrm{kg}$ carvacrol (an inhibitor of TRPM7, Sigma-Aldrich, 282197) in DMSO vehicle daily for 7 consecutive days while the control mice received vehicle alone. At the end of treatment, we used PET/CT to measure the tumors in mice and euthanized them. We dissected, weighed and frozen tumors. Subsequently, we fixed some tumors from each group of mice in $10 \%$ formalin and paraffin-embedded them as well as froze some tumors in liquid nitrogen for subsequent experiments (Figure S5). To perform the PET/CT imaging, we fasted the mice overnight and injected individual mice intravenously with approximately $200 \pm 10 \mu \mathrm{Ci}$ 18-fluoro-6-deoxy-glucose (FDG, Wuhan Union Hospital PET Center, Wuhan, China). One hour later, we anesthetized the mice with $2 \%$ isoflurane and imaged them using PET with a static mode of 10 min and using CT scan of normal mode in the TransPET Discoverist 180 system (Raycan Technology, Suzhou, China). We reconstructed the PET images using the three-dimensional (3D) OSEM method with a voxel size of $0.5 \times 0.5 \times 0.5 \mathrm{~mm} 3$. We reconstructed the CT images using FDK algorithm with $256 \times 256 \times 256$ matrix. We displayed the images with software Carimas (Turku PET Center, Turku, Finland). We calculated the mean standardized uptake value (SUV) using the following formula: mean pixel value with the decay-corrected region-of-interest activity $(\mu \mathrm{Ci} / \mathrm{kg}) /(\mathrm{injected} \mathrm{dose}[\mu \mathrm{Ci}] / \mathrm{weight}[\mathrm{kg}])$. The experimental protocols were approved by the Animal Research and Care Committee of our hospital (approval number: 2019-014).

\section{Cell proliferation}

The cell proliferation was tested by EdU assay, using the BeyoClick ${ }^{\mathrm{TM}}$ EdU-594 Cell Proliferation Assay Kit (Byotime), per the manufacturer's protocol. Briefly, the different groups of cells $\left(3 \times 10^{5}\right.$ cells/well) were cultured in 6-well plates for $48 \mathrm{~h}$ and labeled in triplicate with EdU and DAPI. The fluorescent signals were captured under a fluorescence microscope (Zeiss, Germany).

\section{Flow Cytometry}

The different groups of cells were stained in duplicate using the Annexin V-FITC/PI Apoptosis Detection Kit (Vazyme, Nanjing, China). The frequency of apoptotic cells was mwasured by flow cytometry in the BD Flow Cytometry System.

\section{Intracellular ROS levels}

The contents of intracellular ROS in each group of cells were quantified using Reactive Oxygen Species Assay Kit (Byotime), per the manufacturer's protocol. The frequency of fluorescence positive cells was quantified by flow cytometry using CellQuest software.

\section{Measurement of glucose uptake and lactate production}

The impact of TRPM7 silencing on glucose uptake and lactate production in ieach group of ovarian cancer cells was tested using the glucose uptake colorimetric assay kit and lactate colorimetric assay kit (Biovision, USA), following the manufacturer's protocols.

\section{Seahorse assay}

We performed seahorse assays to quantitatively measure ECAR and OCR of individual groups of cells using Seahorse XF Glycolysis Stress Test Kit and Seahorse XF Cell Mito Stress Test Kit in the Seahorse XFe 96 Extracellular Flux Analyzer (Seahorse Bioscience, USA), following the manufacturer's protocols. Briefly, individual groups of cells $\left(1 \times 10^{4}\right.$ cells/well) were cultured into a Seahorse XF 96-well microplate and their baseline measures were determined. Subsequently, the cells were treated sequentially with glucose, oligomycin (the oxidative phosphorylation inhibitor), and 2-DG (the glycolytic inhibitor) at indicated dose and time points for measurement of ECAR. Similarly, the cells were treated sequentially with oligomycin, oxidative phosphorylation FCCP ( $p$ trifluoromethoxy carbonyl cyanide phenylhydrazone, the reversible inhibitor), and rotenone/antimycin A (Rote/AA, the mitochondrial complex I inhibitor and the mitochondrial complex III inhibitor, respectively). Data were analyzed by Seahorse XF-96 Wave software and expressed as pmols/min for OCR and $\mathrm{mpH} / \mathrm{min}$ for ECAR, respectively.

\section{ATP assay}

The levels of ATP production in each group of cells were determined by ATP assays using ATP Assay Kit (Byotime). Briefly, individual groups of cells were suspended in an ice-cold ATP-releasing buffer, and centrifuged. Individual supernatants or standard (100 $\mu \mathrm{L}$ each) were mixed with $100 \mu \mathrm{L}$ ATP detection solution and analyzed using the Dual-Luciferase Reporter Assay System (Promega). After normalized with protein concentrations, the luminescent signals were used to quantify the levels of ATP, according to the standard curve prepared with known concentrations (1 nM - $1 \mathrm{mM})$ of ATP.

\section{In vivo ubiquitination assays}


The impact of TRPM7 silencing on the stability of HIF-1a in ovarian cancer cells was examined by in vivo ubiquitination assays. Briefly, the different groups of cells were treated in triplicate with $\mathrm{CC}(20 \mu \mathrm{M})$ under a normoxic or hypoxic condition for one day. The cells were treated with MG132 (10 $\mu \mathrm{M})$ for $8 \mathrm{~h}$, and the levels of HIF-1a ubiquitination were determined by IP with anti-HIF-1a antibody, followed by Western blot using anti-Ub antibody (10201-1-AP, Porteintech, $1: 1000)$.

\section{Statistical analysis}

We expressed the data as representative images or the mean \pm SD of each group from three independent experiments. We compared the data from multiple groups using ANOVA and post hoc Bonferroni analysis and the data from two groups by Student's T-test using the SPSS version 18.0 (SPSS, Chicago, IL, USA). We analysed the potential correlation of two variables by Spearman's rank test. We defined statistical significant difference when a $P$-value of $<0.05$ and labelled the significant levels as $* P<0.05,{ }^{*} \mathrm{P}<0.01, * \star * \mathrm{P}<0.001$ in the figures.

\section{Declarations}

\section{Acknowledgements[}

Not applicable.

\section{Authors' contributions[}

Lu Liu and Yongchang Chen performed most of the experiments, analyzed data, and wrote the manuscript. Longzheng Xia, Nayiyuan Wu, Ying Wang, He Li and Xue Chen reviewed and edited the manuscript. Xiaoye Zhang, Zhaoyi Liu and Miaochen Zhu performed some experiments. Jing Wang and Qianjin Liao designed the experiments and edited the manuscript. Jing Wang is the guarantor of this work and has full access to all data in the study and takes responsibility for the integrity of the data and the accuracy of the data analysis. The authors read and approved the final manuscript.

\section{Funding}

This study was supported by grants from National Natural Science Foundation of China (81972836) and Hunan Provincial Natural Science Foundation Science and health joint project(2018JJ6027) and Key R\&D Project of Hunan Provincial Department of Science and Technology(2020SK2120).

\section{Availability of supporting data[}

Not applicable.

\section{Ethical Approval and Consent to participate]}

Informed consent was provided by all patients, and the Joint Ethics Committee of Hunan Cancer Hospital and Affiliated Tumor Hospital of Xiangya Medical College of Central South University of China approved all aspects of this study. For animal studies, all of the experimental procedures were performed in accordance with protocols approved by the Animal Research and Care Committee of Hunan Cancer Hospital.

\section{Consent for publication!}

All authors give consent for the publication of manuscript in Molecular Cancer.

\section{Competing interests}

The authors declare that there is no potential competing interest.

\section{References}

1. Amin, R.W., et al., Patterns of ovarian cancer and uterine cancer mortality and incidence in the contiguous USA. Sci Total Environ, 2019. 697: p. 134128.

2. Torre, L.A., et al., Ovarian cancer statistics, 2018. CA Cancer J Clin, 2018. 68(4): p. 284-296.

3. Allemani, C., et al., Global surveillance of trends in cancer survival 2000-14 (CONCORD-3): analysis of individual records for 37513025 patients diagnosed with one of 18 cancers from 322 population-based registries in 71 countries. Lancet, 2018. 391(10125): p. 1023-1075.

4. Kinnaird, A., et al., Metabolic control of epigenetics in cancer. Nat Rev Cancer, 2016. 16(11): p. 694-707.

5. Hanahan, D. and R.A. Weinberg, Hallmarks of cancer: the next generation. Cell, 2011. 144(5): p. 646-74.

6. Torresano, L., et al., Metabolic reprogramming and disease progression in cancer patients. Biochim Biophys Acta Mol Basis Dis, $2020.1866(5)$ : $\mathrm{p} .165721$.

7. Ma, L. and X. Zong, Metabolic Symbiosis in Chemoresistance: Refocusing the Role of Aerobic Glycolysis. Front Oncol, 2020. 10: p. 5.

8. Li, T., et al., PKM2 coordinates glycolysis with mitochondrial fusion and oxidative phosphorylation. Protein Cell, 2019. 10(8): p. 583-594.

9. Jia, D., et al., Elucidating cancer metabolic plasticity by coupling gene regulation with metabolic pathways. Proc Natl Acad Sci U S A, 2019. 116(9): p. 3909-3918.

10. Yu, L., et al., Modeling the Genetic Regulation of Cancer Metabolism: Interplay between Glycolysis and Oxidative Phosphorylation. Cancer Res, 2017. 77(7): p. 1564-1574. 
11. Yi, Y., et al., Transcriptional suppression of AMPKalpha1 promotes breast cancer metastasis upon oncogene activation. Proc Natl Acad Sci U S A, 2020. 117(14): p. 8013-8021.

12. Jeon, S.M., Regulation and function of AMPK in physiology and diseases. Exp Mol Med, 2016. 48(7): p. e245.

13. Li, S., et al., Ca(2+)-Stimulated AMPK-Dependent Phosphorylation of Exo1 Protects Stressed Replication Forks from Aberrant Resection. Mol Cell, 2019. 74(6): p. 1123-1137 e6.

14. Chauhan, A.S., et al., STIM2 interacts with AMPK and regulates calcium-induced AMPK activation. FASEB J, 2019. 33(2): p. $2957-2970$.

15. Penfold, L., et al., CAMKK2 Promotes Prostate Cancer Independently of AMPK via Increased Lipogenesis. Cancer Res, 2018. 78(24): p. 6747-6761.

16. Salminen, A., K. Kaarniranta, and A. Kauppinen, AMPK and HIF signaling pathways regulate both longevity and cancer growth: the good news and the bad news about survival mechanisms. Biogerontology, 2016. 17(4): p. 655-80.

17. Wang, J., et al., TRPM7 is required for ovarian cancer cell growth, migration and invasion. Biochem Biophys Res Commun, 2014. 454(4): p. 547-53.

18. Liu, L., et al., TRPM7 promotes the epithelial-mesenchymal transition in ovarian cancer through the calcium-related PI3K / AKT oncogenic signaling. J Exp Clin Cancer Res, 2019. 38(1): p. 106.

19. Yang, F., et al., Suppression of TRPM7 Inhibited Hypoxia-Induced Migration and Invasion of Androgen-Independent Prostate Cancer Cells by Enhancing RACK1-Mediated Degradation of HIF-1alpha. Oxid Med Cell Longev, 2020. 2020: p. 6724810.

20. Vander Heiden, M.G. and R.J. DeBerardinis, Understanding the Intersections between Metabolism and Cancer Biology. Cell, 2017. 168(4): p. 657-669.

21. Yang, Y.C., et al., Nuclear translocation of PKM2/AMPK complex sustains cancer stem cell populations under glucose restriction stress. Cancer Lett, 2018. 421: p. 28-40.

22. Madhu, V., et al., Hypoxic regulation of mitochondrial metabolism and mitophagy in nucleus pulposus cells is dependent on HIF-1alpha -BNIP3 axis. J Bone Miner Res, 2020.

23. Zhang, S., et al., Mitochondrial peptide BRAWNIN is essential for vertebrate respiratory complex III assembly. Nat Commun, 2020. 11(1): p. 1312.

24. Moldogazieva, N.T., I.M. Mokhosoev, and A.A. Terentiev, Metabolic Heterogeneity of Cancer Cells: An Interplay between HIF-1, GLUTS, and AMPK. Cancers (Basel), 2020. 12(4).

25. Akimoto, R., et al., DGKzeta depletion attenuates HIF-1alpha induction and SIRT1 expression, but enhances TAK1-mediated AMPKalpha phosphorylation under hypoxia. Cell Signal, 2020. 71: p. 109618.

26. Huang, H., et al., Galangin, a Flavonoid from Lesser Galangal, Induced Apoptosis via p53-Dependent Pathway in Ovarian Cancer Cells. Molecules, 2020. 25(7).

27. Liu, H., J.P. Dilger, and J. Lin, The Role of Transient Receptor Potential Melastatin 7 (TRPM7) in Cell Viability: A Potential Target to Suppress Breast Cancer Cell Cycle. Cancers (Basel), 2020. 12(1).

28. Qiao, W., et al., Effects of Salivary Mg on Head and Neck Carcinoma via TRPM7. J Dent Res, 2019. 98(3): p. 304-312.

29. Wang, J., et al., Overexpression of TRPM7 is associated with poor prognosis in human ovarian carcinoma. Asian Pac J Cancer Prev, 2014. 15(9): p. 39558.

30. Foth, M., et al., FGFR3 mutation increases bladder tumourigenesis by suppressing acute inflammation. J Pathol, 2018. 246(3): p. 331-343.

31. Hsieh, Y.H., et al., Host-derived osteopontin maintains an acute inflammatory response to suppress early progression of extrinsic cancer cells. Int $\mathrm{J}$ Cancer, 2012. 131(2): p. 322-33.

32. Mantovani, A., et al., Neutrophils in the activation and regulation of innate and adaptive immunity. Nat Rev Immunol, 2011. 11(8): p. 519-31.

33. Katz, I.S., et al., 7,12-Dimethylbenz(a)anthracene-induced genotoxicity on bone marrow cells from mice phenotypically selected for low acute inflammatory response. DNA Repair (Amst), 2016. 37: p. 43-52.

34. Cuzzocrea, S., et al., Inducible nitric oxide synthase-knockout mice exhibit resistance to pleurisy and lung injury caused by carrageenan. Am J Respir Crit Care Med, 2000. 162(5): p. 1859-66.

35. Lim, Y., et al., Silica-induced apoptosis in vitro and in vivo. Toxicol Lett, 1999. 108(2-3): p. 335-9.

36. Salvemini, D., et al., Pharmacological manipulation of the inflammatory cascade by the superoxide dismutase mimetic, M40403. Br J Pharmacol, 2001. 132(4): p. 815-27.

37. DeBerardinis, R.J., et al., The biology of cancer: metabolic reprogramming fuels cell growth and proliferation. Cell Metab, 2008. 7(1): p. 11-20.

38. Guppy, M., E. Greiner, and K. Brand, The role of the Crabtree effect and an endogenous fuel in the energy metabolism of resting and proliferating thymocytes. Eur J Biochem, 1993. 212(1): p. 95-9.

39. Zahra, K., et al., Pyruvate Kinase M2 and Cancer: The Role of PKM2 in Promoting Tumorigenesis. Front Oncol, 2020. 10: p. 159.

40. Chen, M., et al., TBC1D8 Amplification Drives Tumorigenesis through Metabolism Reprogramming in Ovarian Cancer. Theranostics, 2019.9 (3): p. 676-690.

41. Shang, Y., et al., CHIP/Stub1 regulates the Warburg effect by promoting degradation of PKM2 in ovarian carcinoma. Oncogene, 2017. 36(29): p. 41914200.

42. Sfakianaki, M., et al., PKM2 expression as biomarker for resistance to oxaliplatin-based chemotherapy in colorectal cancer. Ann Oncol, 2019. 30 Suppl 4: p. iv21-iv22.

43. Xiao, M., et al., The eEF2 kinase-induced STAT3 inactivation inhibits lung cancer cell proliferation by phosphorylation of PKM2. Cell Commun Signal, 2020. 18(1): p. 25.

44. Yamamoto, K., et al., Molecular Imaging of the Tumor Microenvironment reveals the Relationship between Tumor Oxygenation, Glucose Uptake and Glycolysis in Pancreatic Ductal Adenocarcinoma. Cancer Res, 2020.

Page 9/16 
45. Hu, T., et al., Tumor-intrinsic CD47 signal regulates glycolysis and promotes colorectal cancer cell growth and metastasis. Theranostics, 2020. 10(9): $\mathrm{p}$. 4056-4072.

46. Ma, H., et al., c-Src Promotes Tumorigenesis and Tumor Progression by Activating PFKFB3. Cell Rep, 2020. 30(12): p. $4235-4249$ e6.

47. Maeding, N., T. Verwanger, and B. Krammer, Boosting Tumor-Specific Immunity Using PDT. Cancers (Basel), 2016. 8(10).

48. Tyszka-Czochara, M., et al., Caffeic Acid Targets AMPK Signaling and Regulates Tricarboxylic Acid Cycle Anaplerosis while Metformin Downregulates HIF1alpha-Induced Glycolytic Enzymes in Human Cervical Squamous Cell Carcinoma Lines. Nutrients, 2018. 10(7).

49. Zhao, X.L. and C.Z. Yu, Vosaroxin induces mitochondrial dysfunction and apoptosis in cervical cancer HeLa cells: Involvement of AMPK/Sirt3/HIF-1 pathway. Chem Biol Interact, 2018. 290: p. 57-63.

50. Murata, Y., et al., Knockdown of AMPKalpha decreases ATM expression and increases radiosensitivity under hypoxia and nutrient starvation in an SV40transformed human fibroblast cell line, LM217. Biochem Biophys Res Commun, 2018. 495(4): p. 2566-2572.

51. Hu, Y., et al., The AMPK-MFN2 Axis Regulates MAM Dynamics and Autophagy Induced by Energy Stresses. Autophagy, 2020.

52. Zhang, Z., et al., Metformin Enhances the Antitumor Activity of CD8(+) T Lymphocytes via the AMPK-miR-107-Eomes-PD-1 Pathway. J Immunol, 2020.

53. Deng, L., et al., The role of ubiquitination in tumorigenesis and targeted drug discovery. Signal Transduct Target Ther, 2020. 5: p. 11.

54. Li, Y., et al., Dual Roles of the AMP-Activated Protein Kinase Pathway in Angiogenesis. Cells, 2019. 8(7).

55. Jin, K., et al., Activation of AMP-activated protein kinase during sepsis/inflammation improves survival by preserving cellular metabolic fitness. FASEB J, 2020.

56. Yano, N., et al., Irisin counteracts high glucose and fatty acid induced cytotoxicity by preserving AMPK-insulin receptor signaling axis in C2C12 myoblast. Am J Physiol Endocrinol Metab, 2020.

57. Feng, H., et al., Targeting tumor cell-derived CCL2 as a strategy to overcome Bevacizumab resistance in ETV5(+) colorectal cancer. Cell Death Dis, 2020. 11(10): p. 916.

58. Zhao, Q., et al., Targeting Mitochondria-Located circRNA SCAR Alleviates NASH via Reducing mROS Output. Cell, 2020. 183(1): p. 76-93 e22.

\section{Figures}

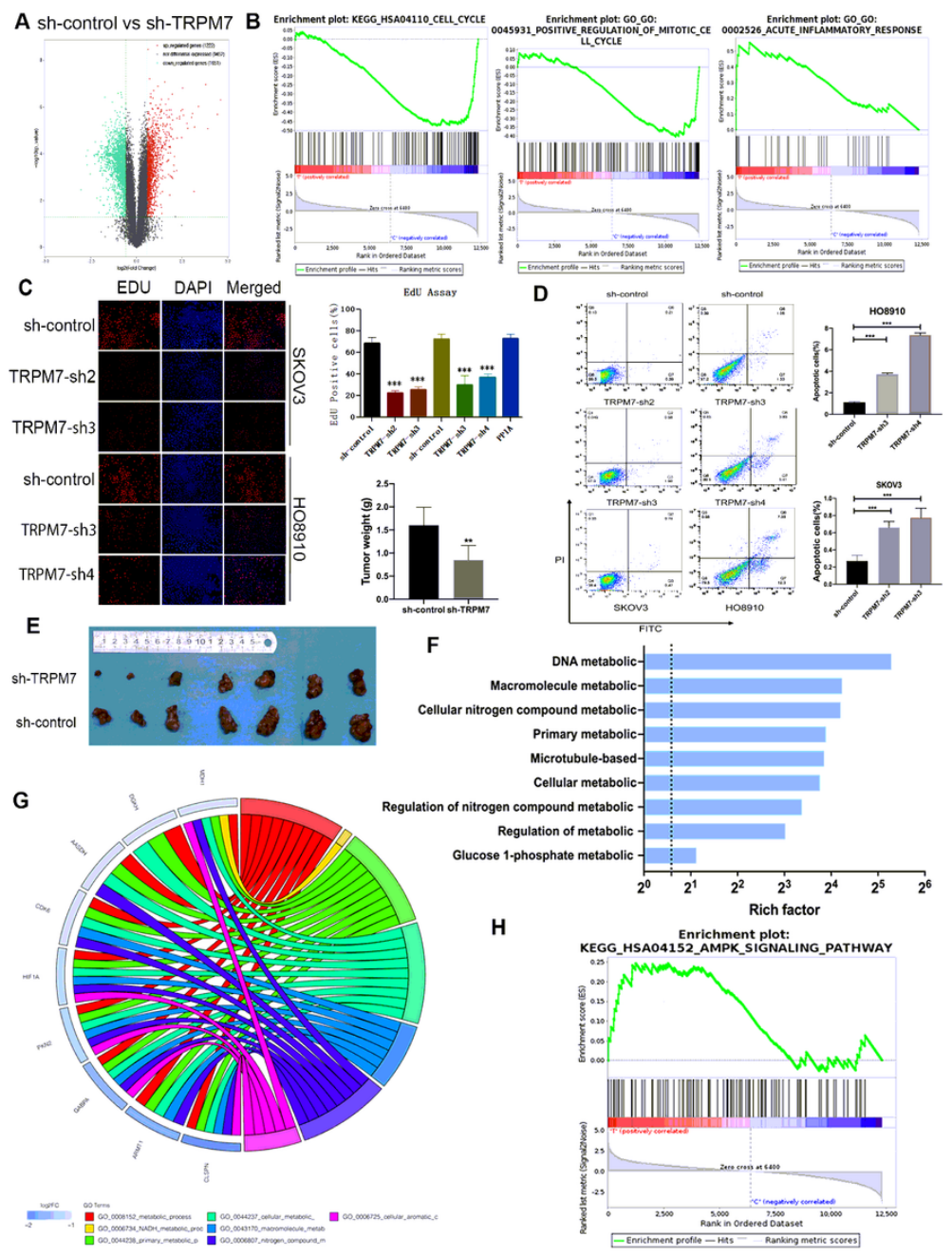

Figure 1 
TRPM7 promotes the growth of ovarian cancer. (A) A volcano plot displayed the DEGs between sh-control SKOV3 and sh-TRPM7 SKOV3 cells. (B) GSEA analysis predicted that TRPM7 was involved in cell cycling and positive regulation of cell mitosis and acute inflammatory. (C) EdU assay exhibited that TRPM7 silencing inhibited cell proliferation. Scale bar $=50 \mu \mathrm{m}$. (D)TRPM7 silencing increased the frequency of apoptotic SKOV3 and HO8910 cells. (E) TRPM7 silencing inhibited the growth of xenograft SKOV3 in vivo. (F) GO analysis exhibited that the DEGs were enriched in the indicated biological processes in the TRPM7 silencing SKOV3 cells. (G) Circos plot displayed the relationships between the DEGs and potential functions in TRPM7 silencing SKOV3 cells.

(H) GSEA analysis predicted that TRPM7 was involved in the AMPK pathway.

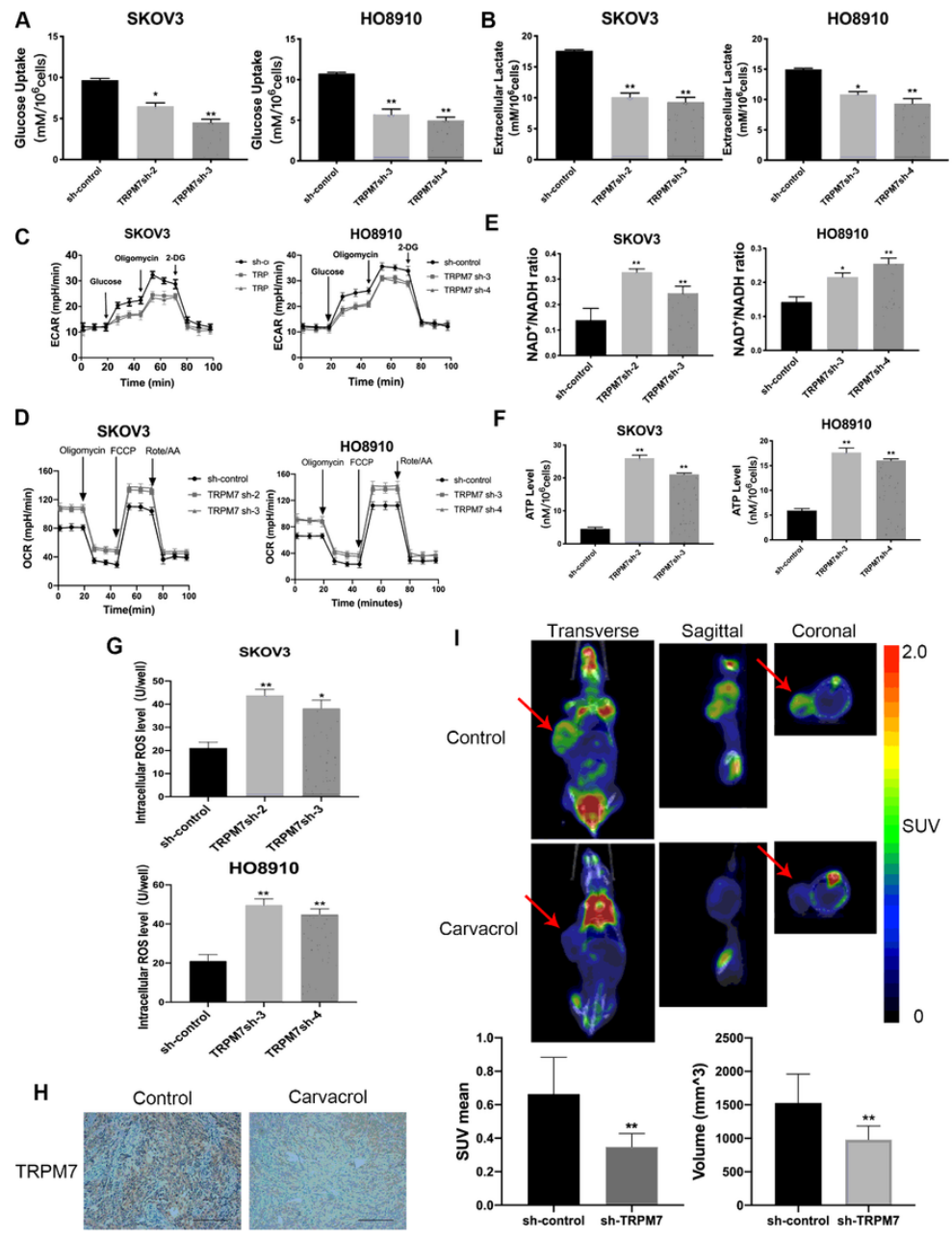

Figure 2

TRPM7 silencing shifts glycolysis to OXPHOS in ovarian cancer cells. (A) The effect of TRPM7 silencing on the glucose uptake levels, (B) extracellular lactate levels, (C) ECARs, (D) NAD+/NADH ratios, (E) intracellular ROS levels, (F) ATP levels, (G) OCRs in SKOV3 and HO8910 cells. (H) IHC analysis of TRPM7 expression in SKOV3 xenograft tumors. Scale bar $=50 \mu \mathrm{m}$. (I) Representative photographs of 18F-FDG PET/CT scans of SKOV3 xenograft tumors in mice following treatment with, or without, carvacrol, a potent inhibitor of TRPM7. SKOV3 and carvacrol were injected to right forelimbs. The SUV max was lower in the carvacrol group than in the control group ( $n=7$ per group). 

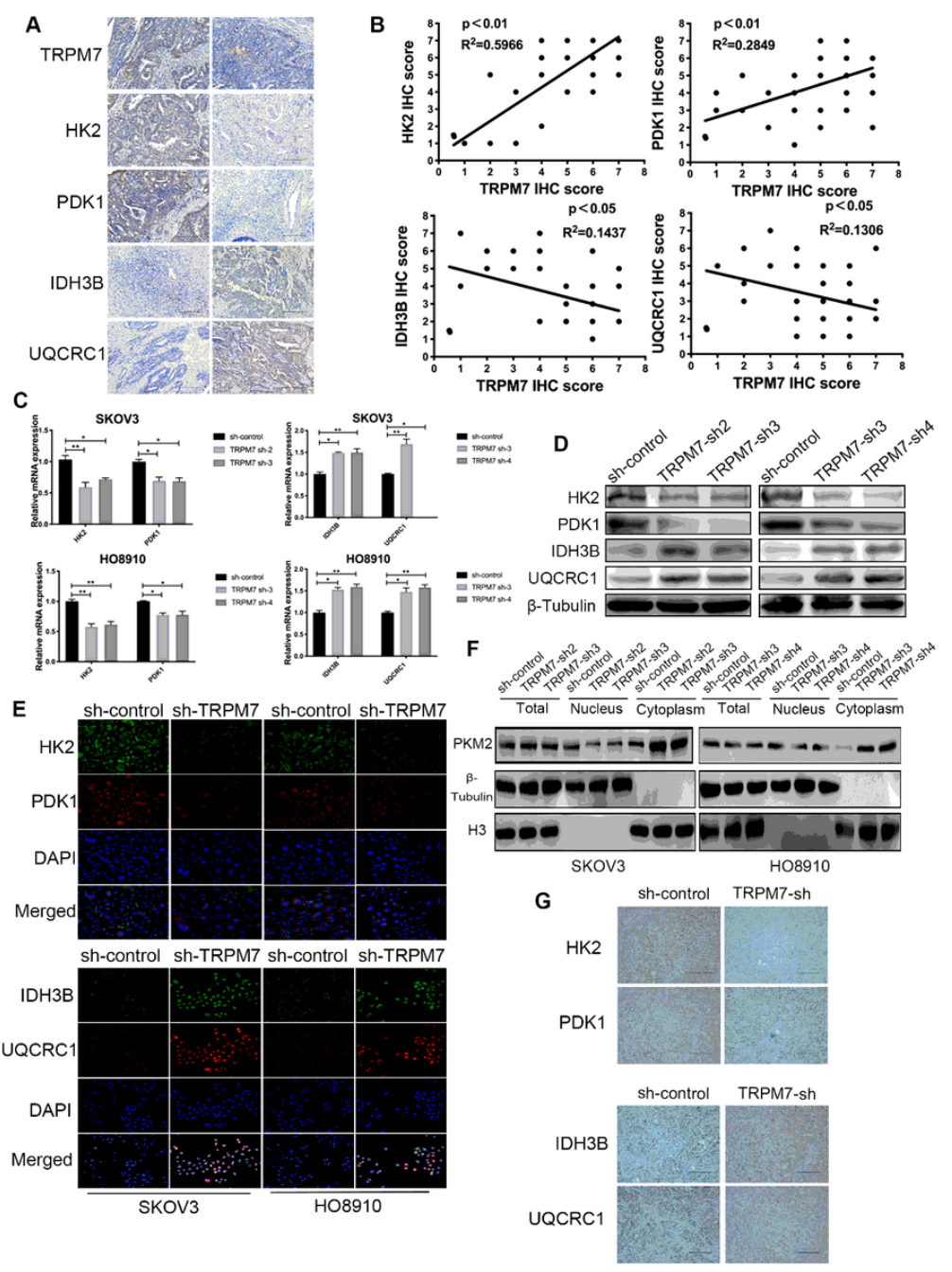

\section{Figure 3}

TRPM7 silencing reduces the expression of glycolysis-related regulators, but enhances the OXPHOS-related regulators in ovarian cancer cells (A) IHC analysis of TRPM7, HK2, PDK1, IDH3B, UQCRC1 expression in ovarian cancer tissues and non-tumor ovarian tissues. Scale bar $=50 \mu \mathrm{m}$. (B) Correlation analysis between the levels of TRPM7 and HK2, PDK1, IDH3B, or UQCRC1 expression in ovarian cancer tissues. (C-D) Western blot and qRT-PCR analyses of HK2, PDK1, IDH3B and UQCRC1 expression in the indicated cells. (E) Immunofluorescent analysis of HK2, PDK1, IDH3B and UQCRC1 expression in ovarian cancer cells after stained with mouse anti-HK2, rabbit anti-PDK1, mouse anti-IDH3B, rabbit anti-UQCRC1 and subsequent Alexa Fluor ${ }^{\text {Tm}} 488$-goat anti-mouse IgG and Alexa Fluor ${ }^{\text {TM }}$ 594-goat anti-rabbit IgG as well as DAPI, scale bar $=50 \mu \mathrm{m}$. (F) Western blot analysis of PKM2 in the nuclear and cytoplasmic fractions following TRPM7 silencing in SKOV3 and H08910 cells. (G) IHC analysis of HK2, PDK1, IDH3B and UQCRC1 expression (scale bar $=50 \mu \mathrm{m})$. 

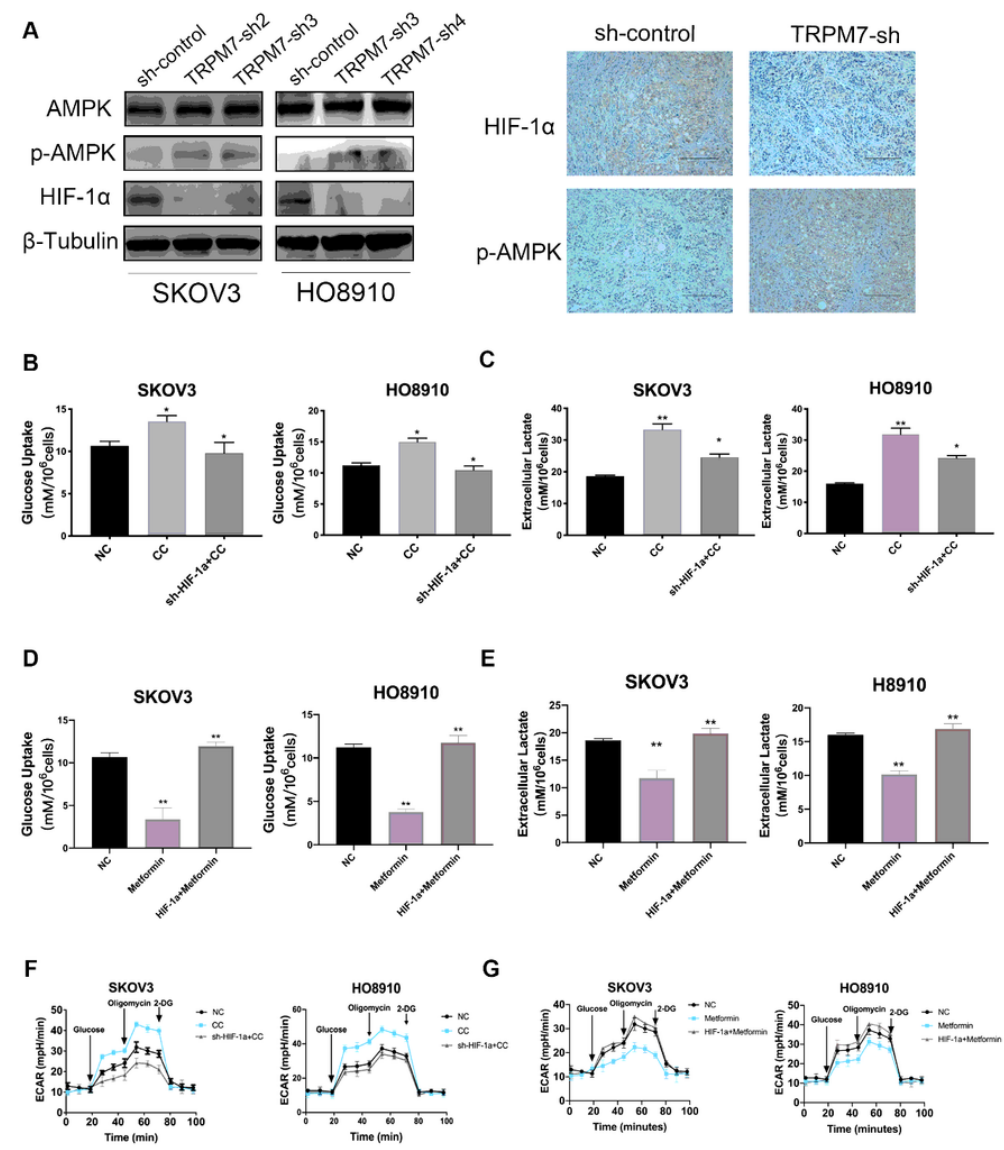

\section{Figure 4}

TRPM7 silencing enhances the AMPK activation, which is associated with inhibiting glycolysis in ovarian cancer cells. (A) Western blot and IHC analyses of HIF-1 a expression and AMPK phosphorylation in the indicated cells and xenograft SKOV3 tumors (scale bar $=50 \mu \mathrm{m}$ ). (B, C, F) Treatment with CC to inhibit AMPK activation enhanced glycolysis, which was mitigated by HIF-1a silencing, by measuring glucose uptake (B), lactic acid production (C) and ECAR (F) in SKOV3-sh-control, SKOV3-sh-TRPM7, H08910-sh-control and H08910-sh-TRPM7 cells. (D, E, G) Treatment with metformin to enhance the AMPK activation attenuated glycolysis, which was abrogated by HIF-1a over-expression, by measuring glucose uptake (D), lactic acid production (E) and ECAR (G) in the indicated cells. 
A
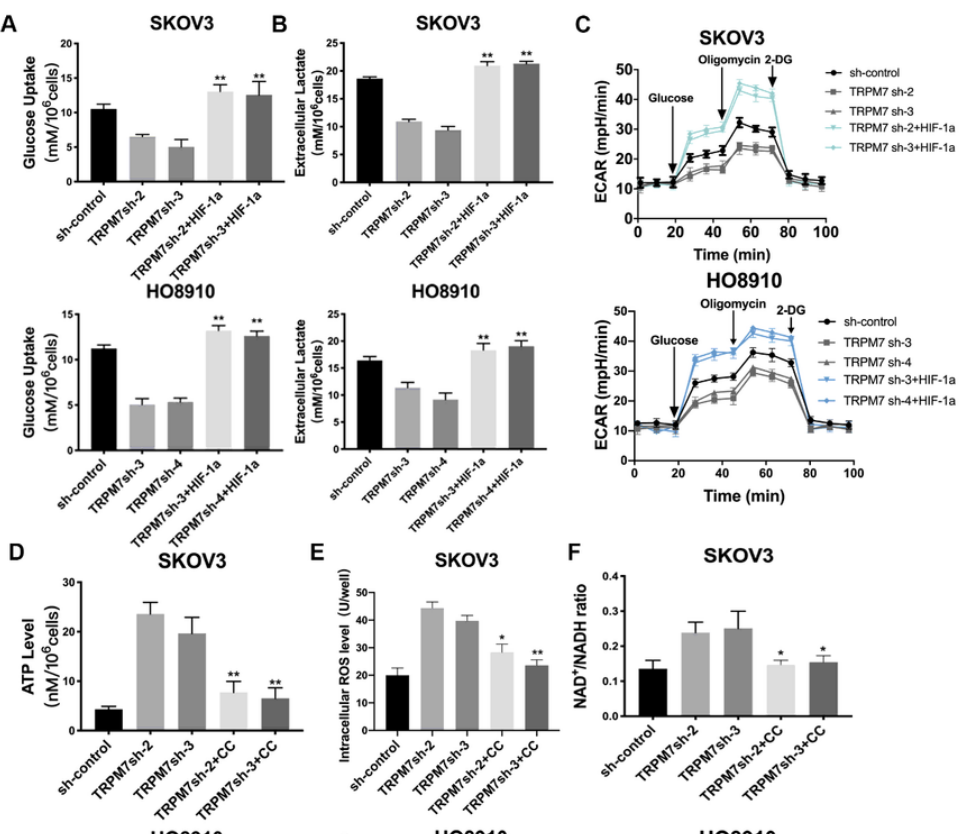

HO8910
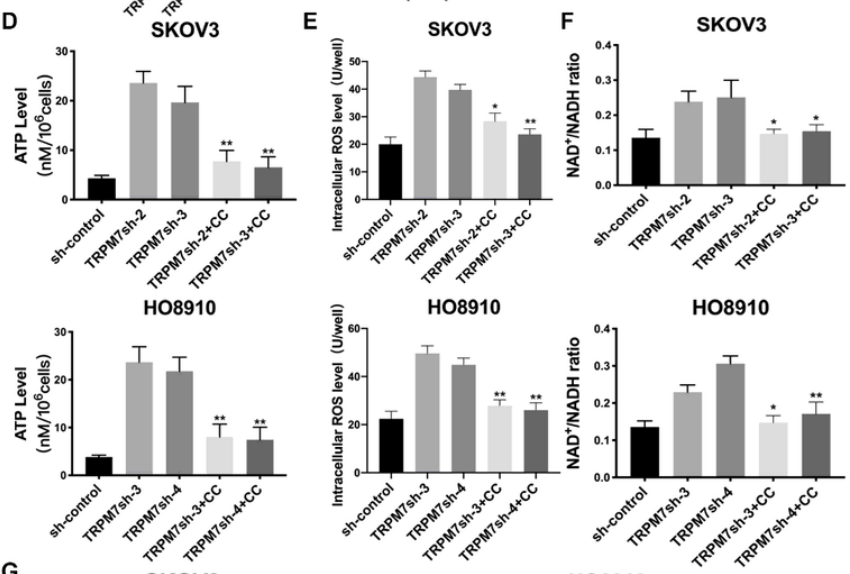

HO8910
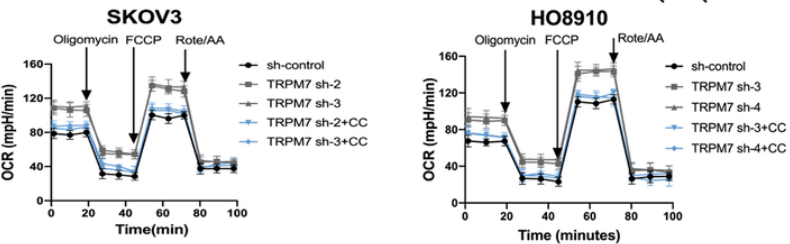

Figure 5

The shifting glycolysis to OXPHOS by TRPM7 silencing is abrogated or mitigated by HIF-1a over-expression and AMPK inhibition in ovarian cancer cells. HIF1a over-expression abrogated the TRPM7 silencing-promoted glycolysis in ovarian cancer cells, determined by the glucose uptake (A), lactic acid production (B) and ECAR (C). Treatment with compound C $(20 \mu \mathrm{M})$ to inhibit AMPK activation mitigated the TRPM7 silencing-induced OXPHOS in ovarian cancer cells, determined by the ATP levels (D), ROS levels (E), NAD+/NAPH rations (F) and OCR (G). 

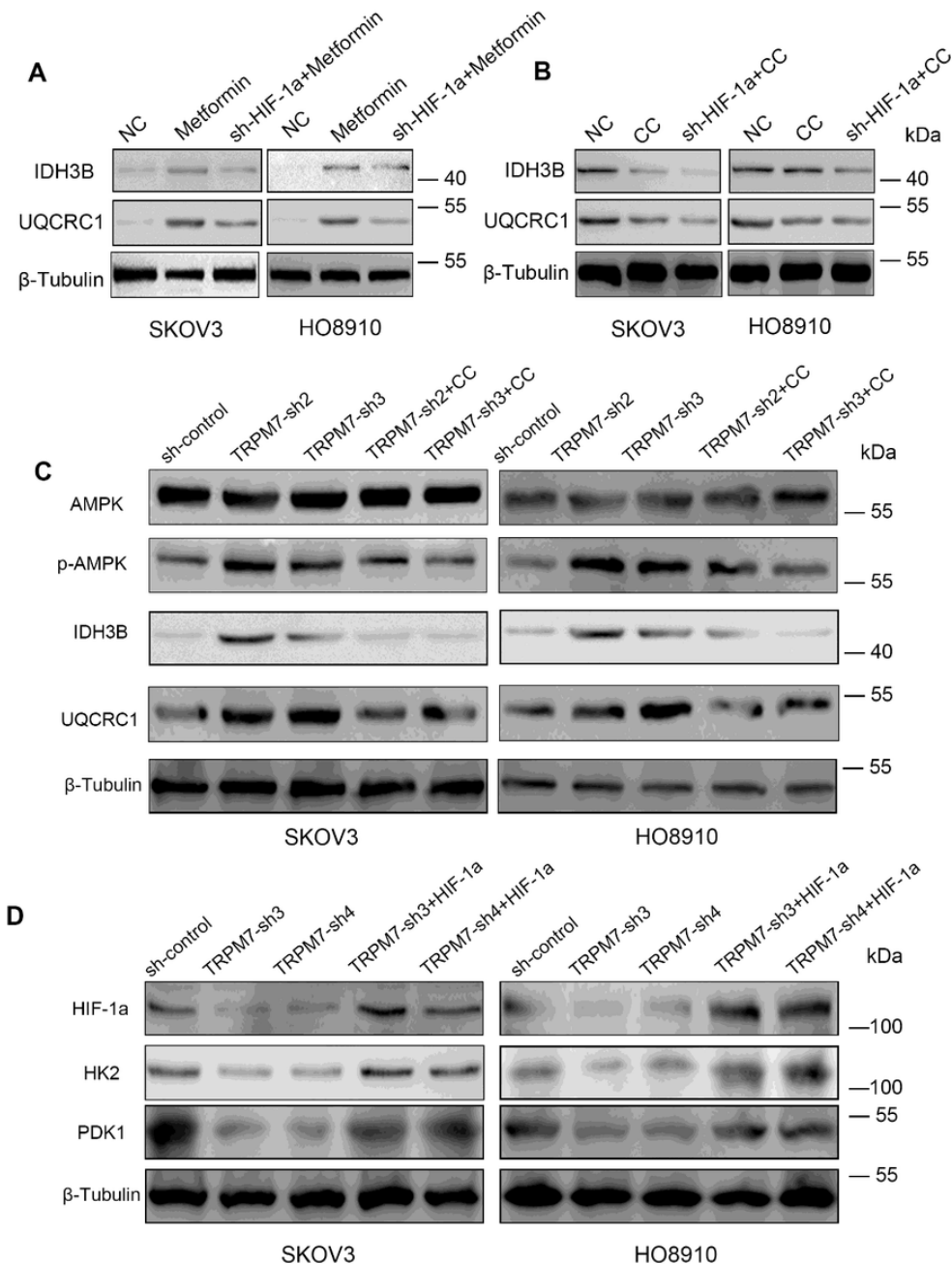

\section{Figure 6}

Altered AMPK activation or HIF-1a expression modulates the TRPM7 silencing-decreased expression of glycolysis-relative regulators and increased expression of OXPHOS-relative regulators in ovarian cancer cells. (A-D) Western blot analysis of IDH3B, UQCRC1, HIF-1a, HK2, PDK1 expression and AMPK phosphorylation in the indicated groups of cells. 


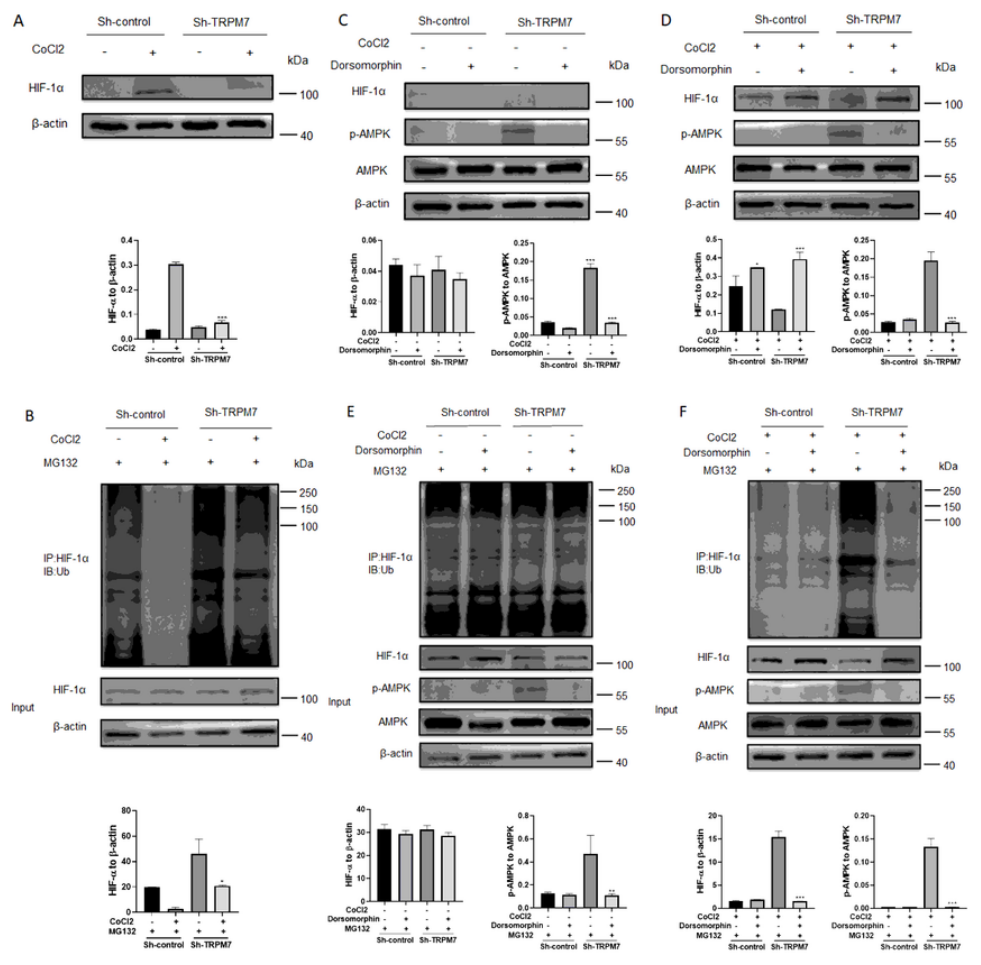

\section{Figure 7}

TRPM7 silencing activates AMPK signaling pathway to promote ubiquitination and degradation of HIF-1a in ovarian cancer cells. (A) Western blot displayed that TRPM7 silencing decreased HIF-1 protein in ovarian cancer cells under a hypoxic condition. (B) In vivo ubiquitination assays indicated that the decreased HIF-1 a protein by TRPM7 silencing was abrogated by treatment with MG132, suggesting that TRPM7 silencing promoted HIF-1a ubiquitination and degradation in SKOV3 cells. (C) There was no detectable HIF-1a protein in the SKOV3 cells under a normoxic condition. (D) Inhibition of AMPK activation by CC increased HIF-1 a protein in both control and TRPM7 silencing SKOV3 cells under a hypoxic condition. (E, F) In vivo ubiquitination assays revealed that treatment with $\mathrm{CC}$ to inhibit AMPK activation mitigated the TRPM7 silencing-induced HIF-1a ubiquitination and degradation in ovarian cancer cells under both normoxic and hypoxic conditions.

\section{Supplementary Files}

This is a list of supplementary files associated with this preprint. Click to download.

- SupplementaryInfo.docx 